\title{
Efficient and Accurate Computation of Eye Diagrams and Bit-Error Rates in a Single-Channel CRZ System
}

\author{
Ronald Holzlöhner, Curtis R. Menyuk, William L. Kath, and Vladimir S. Grigoryan
}

\begin{abstract}
We use linearization to compute the noise evolution in a 10-Gb/s single-channel chirped return-to-zero (CRZ) system propagating over $6100 \mathrm{~km}$, transmitting 32 bits. Linearization allows us to efficiently and accurately compute eye diagrams and bit error rates (BERs) without the use of Monte Carlo simulations. Phase jitter prevents the successful application of linearization unless it is removed, and we describe a method to separately remove it from each pulse in the signal. We show that the BER in our test system is dominated by the bit pattern that leads to the smallest eye opening in a noise-free simulation.
\end{abstract}

Index Terms-Amplifier noise, linear approximation, Monte Carlo methods, optical fiber communication, optical kerr effect, phase jitter, receivers, spectral analysis.

\section{INTRODUCTION}

$\mathbf{N}$ OISE in optical communications systems deteriorates the bit-error-rate (BER) and sets the lower limit on the signal power. BERs are commonly estimated by running Monte Carlo simulations and extrapolating the results under the assumption that the electrical power at the receiver after narrow-band filtering is Gaussian distributed in the marks (ONES) and spaces (ZEROS) [1]. This method is time consuming and not always reliable. In this letter, we report on the application of linearization to calculate the eye diagrams and BER in a single-channel chirped return-to-zero (CRZ) system propagating 32 bits over $6100 \mathrm{~km}$. We focused on this system because it is well-characterized in simulations [2], and it is similar to one of the channels in some commercial wavelength-division-multiplexing (WDM) submarine systems [3]. We have also successfully applied this approach to a CRZ system with multiple channels, and we will report on these results in a future publication.

Previous study of a dispersion-managed soliton (DMS) system [4] showed that it is possible to use linearization to calculate the effects of accumulated noise, but only if the phase and timing jitter are handled separately from the other noise components. This separation is necessary because the nonlinear equations that govern the fiber transmission [4] imply that

Manuscript received February 22, 2002. This work was supported by the Air Force Office of Scientific Research, the Department of Energy, and the National Science Foundation.

R. Holzlöhner is with the Department of Computer Science and Electrical Engineering, University of Maryland Baltimore County, Baltimore, MD 21250 USA (e-mail: holzloehner@umbc.edu).

C. R. Menyuk is with the Department of Computer Science and Electrical Engineering, University of Maryland Baltimore County, Baltimore, MD 21250 USA and also with PhotonEx Corporation, Maynard, MA 01754 USA.

W. L. Kath is with the Department of Engineering Sciences and Applied Mathematics, Northwestern University, Evanston, IL 60208 USA and also with University of Maryland Baltimore County, Baltimore, MD 21250 USA.

V. S. Grigoryan was with University of Maryland Baltimore County, Baltimore, MD 21250 USA and is now with CeLight, Inc., Silver Spring, MD 20904 USA.

Publisher Item Identifier S 1041-1135(02)06083-4. small amounts of amplitude and frequency noise can lead to large amounts of phase and timing jitter respectively, which can invalidate the linearization. However, these nonlinear equations are also phase and time invariant, which implies that phase and timing jitter can be removed from the standard Fourier basis without affecting the subsequent evolution, in which case the coefficients of the modified Fourier basis, along with the phase and timing jitter, remain multivariate-Gaussian distributed far longer than the original Fourier coefficients [4]. ${ }^{1}$ This result is well known in the theory of solitons, where it is standard to use a basis set that consists of discrete as well as continuous components, rather than the usual Fourier basis, when studying the effects of perturbations and noise [5]-[7].

In this letter, we use a direct solution of the linearized evolution equation to obtain the covariance matrix; we previously used Monte Carlo simulations [4]. For the CRZ system that we are studying here, we have found that it is necessary to remove the phase jitter, but not the timing jitter. The basic approach that we use is to follow the standard Fourier basis, projecting out and removing the contribution to the phase from the Fourier coefficients at each amplifier, before the phase fluctuations have had the opportunity to grow beyond the range of validity of the linearization. At the end of the transmission line, there is a square law receiver which is insensitive to the phase, so we do not keep track of it.

We note that our approach assumes that noise-noise interactions are negligible during transmission, but we take them into account in the receiver, which we also assume has a realistic narrow-band electrical Bessel filter. Thus, our work is a generalization of [8], in that the noise that enters the receiver is not white, but is determined by the actual transmission, and we apply realistic electrical filtering.

Our approach is fully deterministic and does not rely at all on Monte Carlo simulations, which allows us to greatly increase the accuracy at small BERs at a fraction of the computational cost. We extend previous work [4] by showing that it is possible to remove the phase jitter from the covariance matrix at each amplifier and for each signal pulse separately, allowing us for the first time to deal with strings containing many overlapping bits. To validate our results, we compare them to standard Monte Carlo simulations.

\section{Simulation Procedure}

Our simulated transmission line consists of 34 dispersion map periods each of length $180 \mathrm{~km}$, for a total distance of $6,120 \mathrm{~km}$. Each map period contains a $160-\mathrm{km}$ span of normal dispersion

\footnotetext{
${ }^{1}$ We note that the phase is only Gaussian distributed if we track the phase change on the infinite line. If we only track it in the range $[0,2 \pi]$, then it is Jacobi- $\Theta$ function distributed. This function is the periodic analogue of a Gaussian distribution.
} 
fiber with $-2.5 \mathrm{ps} / \mathrm{nm} \cdot \mathrm{km}$, followed by a $20-\mathrm{km}$ span of anomalous dispersion fiber with $16.5 \mathrm{ps} / \mathrm{nm} \cdot \mathrm{km}$. We use precompensating and postcompensating fibers, each of which has a total dispersion of $916 \mathrm{ps} / \mathrm{nm}$. The loss is compensated every $45 \mathrm{~km}$ by an erbium-doped fiber amplifier (EDFA) with a spontaneous emission factor of $n_{\mathrm{sp}}=2.0$. The signal pulses are copolarized and have a full-width at half-maxiumu (FWHM) duration of 28 ps with a chirped raised-cosine shape of the form

$u(t)=\left(\frac{1}{2} P_{\text {peak }}\left\{1+\cos \left[\pi \sin \left(\frac{\Omega t}{2}\right)\right]\right\}\right)^{1 / 2}$

$\times \exp (i A \pi \cos \Omega t)$

where $u(t)$ is the envelope of the optical field at time $t, \Omega=$ $2 \pi / T_{\text {bit }}$ with the bit spacing $T_{\text {bit }}=100$ ps and the chirp parameter is $A=-0.6$ [9]. The initial optical peak power is $P_{\text {peak }}=1 \mathrm{~mW}$ before entering the precompensating fiber. We transmit 32 bits, corresponding to a pseudorandom bit sequence of $2^{5}-1=31$ bits plus an additional zero bit, thereby exhausting all possible bit patterns of length five. Fig. 1 shows the narrow-band filtered noise-free receiver voltage after $6100 \mathrm{~km}$ of transmission.

We proceed by first expressing the optical field envelope as $u=u_{0}+\delta u$, where $u_{0}=\langle u\rangle$ is the noise-free field and $\delta u$ represents accumulated noise. We next write $\delta u=\sum_{k=-N_{\mathrm{FFT}} 2}^{N_{\mathrm{FFT}} / 2-1}\left[\alpha_{k}+i \beta_{k}\right] \exp \left(i \omega_{k} t\right)$, where $\alpha_{k}$ and $\beta_{k}$ represent the $N_{\mathrm{FFT}}$ real and imaginary noise Fourier coefficients and $\omega_{k}=2 \pi k / T$ with the period $T=32 T_{\mathrm{bit}}$. We define the real vector

$$
\begin{aligned}
\boldsymbol{a}=\left(\alpha_{-N / 2}, \alpha_{-N / 2+1}, \ldots,\right. & \alpha_{N / 2-1}, \\
& \left.\beta_{-N / 2}, \beta_{-N / 2+1}, \ldots, \beta_{N / 2-1}\right)^{T}
\end{aligned}
$$

of length $2 N$, where the symbol $T$ denotes the transpose. The vector $\boldsymbol{a}$ spans the low frequency modes from $k=-N / 2$ to $k=N / 2-1$ and we choose $N_{\mathrm{FFT}}=2048$ and $N=140$ in this work. The evolution of the $2 N \times 2 N$ noise covariance matrix $\mathcal{K}=\left\langle\boldsymbol{a} \boldsymbol{a}^{T}\right\rangle$ over one fiber leg from $z=0$ to $z=L$, in which no noise is added, followed by an EDFA with gain $G$, is given by

$$
\mathcal{K}(L)=G \Psi \mathcal{K}(0) \Psi^{T}+\eta \mathcal{I}
$$

where $\Psi$ is a propagator matrix, $\mathcal{I}$ is the identity matrix, and $\eta$ equals half the average amplified spontaneous emission (ASE) noise power per frequency mode. We choose a perturbative method to compute $\Psi$. We first let $u_{0}(t, 0)$ and $u_{0}(t, L)$ be the noise-free optical field at the beginning and end of the fiber span, respectively. We then perturb $u_{0}(t, 0)$ in a single frequency mode $k$ by a small amount $\Delta$ and launch the perturbed signal $u^{(k)}(t, 0)=u_{0}(t, 0)+\Delta \exp \left(i \omega_{k} t\right)$. At $z=L$, we obtain $u^{(k)}(t, L)$ by solving our nonlinear transmission equation and calculate the deviation $\delta u^{(k)}=u^{(k)}(t, L)-u_{0}(t, L)$ and its Fourier space vector $\boldsymbol{a}^{(k)}$. The elements of $\Psi$ are given by $\Psi_{j k}=a_{j}^{(k)} / \Delta$. We find that the perturbation method is numerically stable and its result are independent of the value of $\Delta$ over several orders of magnitude. By successive application of (1), we can propagate the covariance matrix from amplifier to amplifier.

The phase jitter separation is a crucial step, and its application to the present system is the key new contribution of this letter. We define the average phase of a pulse that is confined to a bit slot beginning at $t_{0}$ as
$\varphi=\int_{t_{0}}^{t_{0}+T_{\mathrm{bit}}} \arg [u(t)]|u|^{2} d t / \int_{t_{0}}^{t_{0}+T_{\mathrm{bit}}}|u|^{2} d t$ [4]. If the noise is small, then the component of the noise vector $\boldsymbol{a}^{(k)}$ that will rotate the phase of the signal $\varphi$ is proportional to $\boldsymbol{v}$, where $\boldsymbol{v}$ is the real Fourier expansion vector of $i u_{0}(t, z)$ defined analogously to $\boldsymbol{a}$. We remove the phase jitter that was produced in the fiber span by projecting it out of $\boldsymbol{a}^{(k)}$ to obtain a residual noise vector $\widetilde{\boldsymbol{a}}^{(k)}$ defined by

$$
\widetilde{\boldsymbol{a}}^{(k)}=\boldsymbol{a}^{(k)}-\frac{\left(\boldsymbol{a}^{(k)}, \boldsymbol{v}\right)}{(\boldsymbol{v}, \boldsymbol{v})} \boldsymbol{v}
$$

where $(\cdot, \cdot)$ denotes the scalar product between real vectors. The vector $\widetilde{\boldsymbol{a}}^{(k)}$ is now used instead of $\boldsymbol{a}^{(k)}$ to compute $\Psi$, and hence the phase jitter that is produced during the propagation is no longer included in the covariance matrix $\mathcal{K}$.

Each pulse in the signal has a different phase $\varphi$. In a system in which pulses do not overlap, such as a soliton system, these pulse phases evolve independently and must be removed separately from each other. To deal with nonoverlapping pulses, (2) can be generalized by decomposing the signal $u_{0}(t)$ into a sum of mutually orthogonal pulses, that is $u_{0}(t)=\sum_{l} u_{l}(t)$, where $u_{l}(t)=u_{0}(t)$ for $(l-1) T_{\mathrm{bit}} \leq t<l T_{\mathrm{bit}}$, and $u_{l}(t)=0$ otherwise. Then the vectors $\boldsymbol{a}^{(k)}$ and $\boldsymbol{v}$ are computed for each $u_{l}$ separately and (2) is applied.

In the present system, the maximum FWHM pulse duration is $210 \mathrm{ps}$, leading to a significant overlap of adjacent pulses. We find that the phase jitter can still be removed separately for each pulse after applying artificial dispersion compensation. We pass a copy of the signals $u_{0}(t, L)$ and $u^{(k)}(t, L)$ through an ideal linear and lossless fiber whose total dispersion is $-D(L)$, where $D(L)$ is the total accumulated dispersion at the point $L$ in the transmission system. This procedure separates the pulses and is fully reversible [2]. Then we compute $\widetilde{\boldsymbol{a}}^{(k)}$ by applying (2) to each of the pulses separately and send the signal corresponding to $\widetilde{\boldsymbol{a}}^{(k)}$ back to the point $L$ through an ideal fiber with total dispersion $+D(L)$. One might argue that the phases of overlapping pulses will not evolve independently, and hence might become correlated; however, we find by comparison to Monte Carlo simulations that the procedure just described leads to accurate BERs in the present system, indicating that the coupling of the signal in one pulse to the phase jitter in an overlapping pulse is negligible.

We now summarize the algorithm for propagating $\mathcal{K}$ over one fiber span from $z=0$ to $z=L$, followed by an EDFA. First, propagate the field $u_{0}(t, 0)$ using a standard Fourier split-step algorithm, yielding $u_{0}(t, L)$. Then return to $z=0$ and repeat the following for each $k$ :

1. compute $u^{(k)}(t, L)$ by perturbing $u_{0}(t, 0)$ in the $k$-th frequency mode and propagating it to $z=L$;

2. separate the pulses in the signal by passing $u^{(k)}(t, L)$ through a linear and

lossless fiber with total dispersion $-D(L)$;

3. compute the perturbation vector $\boldsymbol{a}^{(k)}$ and apply the phase jitter removal (2) separately to each pulse, yielding the vector $\widetilde{\boldsymbol{a}}^{(k)}$ of the dispersion compensated signal;

4. invert step 2 to compute $\widetilde{\boldsymbol{a}}^{(k)}$ at point L. Evaluate the propagator matrix elements $\Psi_{j k}$. 


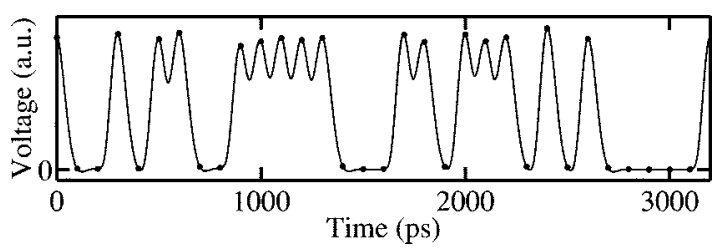

Fig. 1. Narrow-band filtered noise-free receiver voltage of the signal at $6100 \mathrm{~km}$. The $32 \mathrm{pdfs}$ are computed at the points in time indicated by the dots.

Finally, compute $\mathcal{K}(L)$ according to (1).

At the receiver, we assume that there is an ideal square law detector followed by a fifth-order Bessel filter with a FWHM bandwidth of $8.6 \mathrm{GHz}$. In this case, the receiver will produce a generalized chi-square distribution for both the marks and the spaces [4].

\section{RESULTS}

We compute a pdf of the narrow-band filtered receiver voltage at the center of each bit slot as indicated in Fig. 1 by the dots in the noise-free signal. The variation in the peak power is due to nonlinear pulse-to-pulse interactions during the transmission, highlighting the importance of bit patterns.

Fig. 2(a) shows the average pdfs of the receiver voltage that result from the linearization approach as solid lines in comparison with a histogram from a traditional Monte Carlo simulation, consisting of 86000 noise realizations represented by the dots. The voltage is normalized to the mean of the pdf of the marks. The dashed lines show a Gaussian fit to the Monte Carlo data, using the mean and variance. The large deviation between the solid and dashed curves is obvious, especially in the spaces. On the other hand, the agreement between the linearization approach and the Monte Carlo results is excellent. By integrating the pdfs we obtain optimal BERs of $1.7 \times 10^{-12}$ from the linearization approach and $9.9 \times 10^{-12}$ from the Gaussian fit of the Monte Carlo data; the latter corresponds to a $Q$-factor of 6.71 . Note that the relatively small difference between these two BERs occurs because the Gaussian fit overestimates the pdf of the marks and underestimates it in the spaces. The dash-dotted line shows the pdf of the marks that we obtain if we do not separate the phase jitter, which is clearly wrong.

The left solid curve in Fig. 2(b) shows the error probability $P_{1 \mid 0}$ of detecting a "1," using a given decision level, when a " 0 " was sent, corresponding to the left pdf in Fig. 2(a), and the right solid curve is the probability $P_{0 \mid 1}$ of detecting a " 0 " when a "1" was sent. The dashed lines show the same, except that only the mark with the lowest voltage in the noise-free signal and the space with the highest voltage were taken into account, respectively. The error rates near the optimum decision level are dominated by the worst mark and space, respectively. This result indicates that in our system it is sufficient to apply the linearization method only to the patterns that exhibit the worst behavior in the absence of noise to obtain a good approximation of the average pdfs and the BER.

\section{CONCLUSION}

We applied the deterministic noise linearization method to a $10-\mathrm{Gb} / \mathrm{s}$ single-channel CRZ system with a transmission distance of $6100 \mathrm{~km}$. Extending previous work [4], we were able

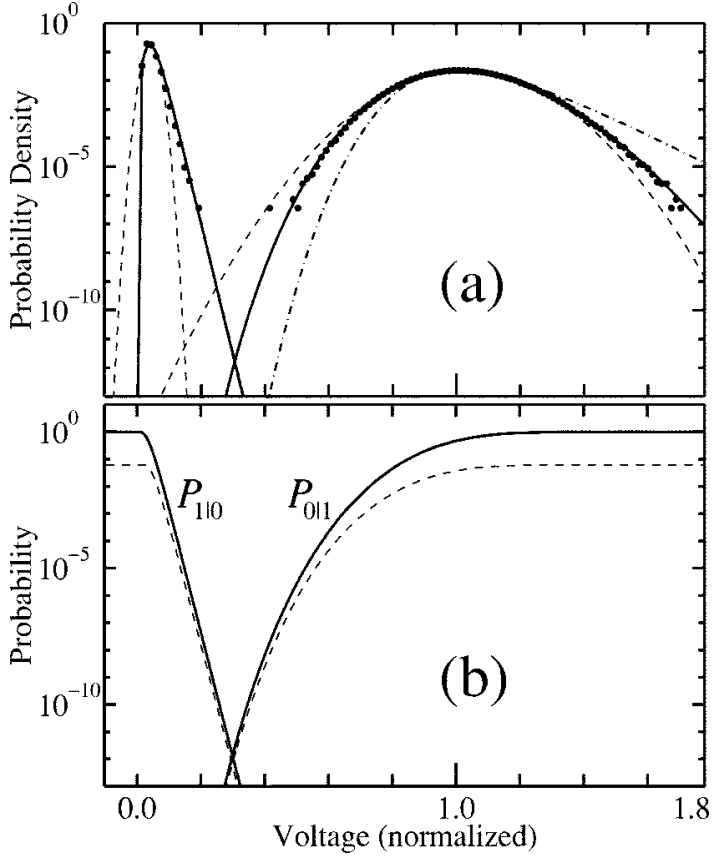

Fig. 2. (a) Solid lines: Average pdfs from the linearization approach. Dots: Histogram from a Monte Carlo simulation. Dashed lines: Gaussian fit to the dots using the mean and variance. Dash-dotted line: Average pdf from the linearization approach without phase jitter separation. (b) Solid lines: Error probabilities $P_{1 \mid 0}$ and $P_{0 \mid 1}$ corresponding to the solid lines in (a). Dashed lines: $P_{1 \mid 0}$ and $P_{0 \mid 1}$ for the worst noise-free mark and space only.

to compute the accurate pdfs and the BER of a signal with 32 strongly overlapping bits. The crucial step in the linearization is the removal of the phase jitter, which must be performed separately for each pulse. Our results also indicate that at least in the present test system, it is sufficient to apply the linearization to the worst noise-free patterns in a bit string which significantly reduces the computational cost of the simulation.

\section{REFERENCES}

[1] N. S. Bergano, F. W. Kerfoot, and C. R. Davidson, "Margin measurements in optical amplifier systems," IEEE Photon. Technol. Lett., vol. 5, pp. 304-306, Mar. 1993.

[2] R.-M. Mu and C. R. Menyuk, "Symmetric slope compensation in a long-haul WDM system using the CRZ format," IEEE Photon. Technol. Lett., vol. 13, pp. 797-799, Aug. 2001

[3] M. Vaa, B. Bakhshi, E. A. Golovchenko, Y. Chai, F. Heismann, H. Li, M. Arend, W. W. Patterson, A. L. Simons, G. T. Harvey, R. L. Maybach, and N. Bergano, "Demonstration of a $640 \mathrm{Gbit} / \mathrm{s} \times 7000 \mathrm{~km}$ submarine transmission system technology ready for field deployment," in Conf. Proc. OFC'01, Anaheim, CA, 2001, Paper WF5.

[4] R. Holzlöhner, V. S. Grigoryan, C. R. Menyuk, and W. L. Kath, "Accurate calculation of eye diagrams and bit error rates in long-haul transmission systems using linearization," J. Lightwave Technol., vol. 20, pp. 389-400, Mar. 2002.

[5] H. A. Haus, "Quantum noise in solitonlike repeater systems," J. Opt. Soc. Amer. B, vol. 8, pp. 1122-1126, 1991.

[6] D. J. Kaup, "Perturbation theory for solitons in optical fibers," Phys. Rev. A, vol. 42, pp. 5689-5694, 1990.

[7] T. Georges, "Perturbation theory for the assessment of soliton transmission control," Opt. Fiber Technol., vol. 1, pp. 97-116, 1995.

[8] D. Marcuse, "Derivation of analytical expressions for the bit-error probability in lightwave systems with optical amplifiers," J. Lightwave Technol., vol. 8, pp. 1816-1823, Dec. 1990.

[9] T.-S. Yang, W. L. Kath, and S. G. Evangelides Jr, "Optimal prechirping for dispersion-managed transmission of return-to-zero pulses," in Conf. Proc. OFC'99, Washington, DC, Paper ThQ4. 